\title{
Research on Population Development Trend in Huizhou of China Forecast Based on Optimal Weighted Combination Method and Fractional Grey Model
}

\author{
Dewang Li $\left(\mathbb{D},{ }^{1}\right.$ Jianbao Chen $\mathbb{D}^{1},{ }^{2}$ and Meilan Qiu $\mathbb{D I}^{1}$ \\ ${ }^{1}$ School of Mathematics and Statistics, Huizhou University, Huizhou 516007, China \\ ${ }^{2}$ School of Mathematics and Statistics, Fujian Normal University, Fuzhou 350117, China \\ Correspondence should be addressed to Jianbao Chen; jbchen@fjnu.edu.cn
}

Received 27 August 2021; Accepted 1 October 2021; Published 3 November 2021

Academic Editor: NDOLANE SENE

Copyright (c) 2021 Dewang Li et al. This is an open access article distributed under the Creative Commons Attribution License, which permits unrestricted use, distribution, and reproduction in any medium, provided the original work is properly cited.

In this paper, the optimal weighted combination model and fractional grey model are constructed. The coefficients of the optimal weighted combination model are determined by minimizing the sum of squares of resists of each model. On the other hand, the optimal conformable fractional order and dynamic background value coefficient are determined by the quantum inspired evolutionary algorithm (QIEA). Taking the resident population from 2008 to 2018 as the research object, the optimal weighted combination model and fractional grey model were used to study the estimated and predicted values. The results are compared and analyzed. The results show that the fractional grey model is better than the optimal weighted combination model in the estimation of the values. The optimal weighted combination model is better than the fractional grey model in predicting. Meanwhile, the fractional grey model is found to be very suitable for the data values that are large, and the changes between the data are relatively small. The research results expand the application of the fractional grey model and have important implications for the policy implementation activities of Huizhou government according to the population growth trend in Huizhou.

\section{Introduction}

With the massive urbanization of the population, population development is still an important issue in China. The current situation of population development is that the proportion of the population with young children is gradually decreasing, and the proportion of the elderly is gradually increasing [1]. Population scale is one of the main indicators of regional economic development, and the population problem is also the basic problem that people pay most attention to. Professor Zeng [2] has analyzed the past, present, and future of demography. Therefore, China has implemented a new birth policy. From the implementation of the "two-child" birth policy in 2013 to the implementation of the "two-child comprehensive" birth policy in 2015, new changes have taken place in population development. Before 2000, the foundation of population and Family Planning in Huizhou was not very good. Huizhou's population reproduction is "high birth rate, low death rate, and high natural population growth rate." The birth rate and natural growth rate show a characteristic fluctuation trend. In 1979, the birth rate was as high as 26.68 per thousand, the death rate remained stable at around 5 per thousand, and the population grew rapidly [3]. In recent years, population development has become a new research hotspot.

The mathematical method of population forecast set the advantage of the statistical method and demography method [4]. In the seventeenth century, there were studies on population prediction abroad, and a series of population prediction methods were summarized. Fitting the population development trend, we can forecast the future population scale development trend according to the geometric progression growth. For example the retardation growth model sets the maximum population carrying capacity under certain environmental conditions. When the population growth reaches the limit, the population growth 
rate is zero. The Leslie model uses the difference equation matrix to discuss the law of population change under the condition of stable development. One is to use statistical methods to build regression models and time series models for population forecasting. The other is the population prediction method, which uses birth, death, and migration data to explore population trends. For example, the cohort factor method sets parameters such as death, birth, and migration and structural change according to the input parameters of population size. Others are the BP neural network method which simulates the biological neural dynamic principle to predict the population with high precision.

In domestic population studies, professor Zha [5] proposed population projections with three possibilities. In the population prediction method, based on cybernetics, Professor Song et al. [6] introduced the birth rate, death rate, fertility rate, and other indicators to construct the population control partial differential equation of population prediction. In the short term forecast, Professor Deng [7] established a grey system to be used in population forecasting. He regarded the population system as a grey system based on model uncertainty and model mathematics, which could obtain more accurate prediction results without too much basic data.

Some Chinese scholars have explored the improvement methods based on the domestic and foreign population prediction models. Chen [8] established the progressive population development model of children's subclass age and made a new attempt to predict population based on demographic structure factors. Hao and Wang constructed a grey dynamic model of China's population development trend and verified the practicability of the grey model [9]. Zhai et al. [10] studied the application of the queuing factor method in population prediction software PADIS-INT and considered that the software has the characteristics of multiple functions, convenient input of parameters, strong applicability, and detailed results, which can be used for more detailed population prediction. Wang [11] derived the problem of progressive fertility parameters in the progressive child population prediction model in detail and proved the practicality of the progressive child population prediction model in population prediction. Wang [11] deduced in detail the problem of the progressive fertility parameters in the progressive child population prediction model and proved the practicability of the progressive child population prediction model in population prediction. To sum up, most of the literature is about the population situation of a country, a province, or a region, while there are few studies on the population development trend of second- and third-tier cities. The combined model can make up for the shortcomings of the single model and can absorb the advantages of the single model. Compared with single model, it has higher precision and predictive value and has higher precision. As far as we know, few scholars use the fractional grey model to study the population situation of prefecture-level cities. Based on this, this paper develops the research and obtains good results. The results of the fractional grey model and the optimal weighted combination model are compared and analyzed. At the same time, this research process is also the attempt to examine the seventh national Population Census Data and statistical yearbook data in Huizhou.

\section{The Optimal Weighted Combination Model}

To extract the most available information, various models are combined to improve the reliability of the prediction. The combination forecasting method is to combine different forecasting submodels into one model. Compared with the single forecasting model, the combined forecasting model is more specific, detailed, comprehensive, and scientific, which can reduce the influence of some random factors on the single forecasting model [12]. The most important part of combinatorial forecasting method is to obtain the coefficient of each submodel, that is, the combination weight coefficient. The estimation of weight coefficient includes optimal weight method, variance-covariance method, recursive variance method, and positive weight synthesis method. In this paper, the optimal weighting method is used. The optimal weighting method is realized by minimizing the total error of the combined prediction in a certain time. The objective function constructed according to a given criterion is minimized under a series of constraints to obtain the weight coefficient [13]. The construction of the objective function depends on the selection of error statistics and the type of minimization criteria.

Suppose $\left\{y_{t}\right\}(t=1,2, \ldots, n)$ for the original data sequence, the original data sequence is first built, and the individual $m$ models are predicted, and the predicted value is $\left\{\hat{y}_{i t}\right\}, i=1,2, \ldots, m$ and $t=1,2, \ldots, n$. And then, the individual $m$ models are combined to calculate combined $K=$ $\left(k_{1}, k_{1}, \ldots, k_{m}\right)^{T}$ combined to calculate combined. The model is the optimal weighted combination model when the statistical error of the model is the minimum, that is, $Q=$ $\sum_{i=1}^{m} e_{i t}^{2}$ the minimum [14]:

$$
\begin{cases}\min & Q, \\ \text { s.t. } & \sum_{i=1}^{m} k_{i}=1,\end{cases}
$$

where $Q$ is the objective function constructed by the model and s.t. is the constraint premise of the objective function. Turn it into a mathematical programming problem as follows:

$$
\begin{cases}\min & Q=\sum_{i=1}^{\mathrm{m}} e_{i t}^{2}=e^{T} e=K^{T} E K, \\ \text { s.t. } & \sum_{i=1}^{m} k_{i}=R^{T} K=1 .\end{cases}
$$

In the formula, $R=[1,1, \ldots, 1]^{T}$ is the matrix of $n \times$ 1 and $E$ is the matrix of estimated error information. Using the Lagrange multiplier formula, from

$$
\frac{\partial}{\partial K}\left(K^{T} E K-2 \lambda\left(R^{T} K-1\right)\right)=0,
$$


we obtain

$$
\begin{aligned}
E K-\lambda R & =0, \\
K & =\lambda E^{-1} R .
\end{aligned}
$$

At the same time, from

$$
\frac{\mathrm{d}}{\mathrm{d} \lambda}\left(K^{T} E K-2 \lambda\left(R^{T} K-1\right)\right)=0,
$$

we obtain

$$
\begin{aligned}
R^{T} K & =1, \\
R^{T} \lambda E^{-1} R & =1, \\
\lambda & =\frac{1}{R^{T} E^{-1} R} .
\end{aligned}
$$
solved:

Combining (4) and (6), the optimal weighted vector is

$$
K_{0}=\frac{E^{-1} R}{R^{T} E^{-1} R} .
$$

The minimum value of the objective function is as follows:

$$
Q_{0}=K_{0}^{T} E K_{0}=\frac{1}{R^{T} E^{-1} R} .
$$

\section{The Fractional Grey Model}

3.1. FGM $(1,1)$ Mode. The fractional grey model is firstly propounded by $\mathrm{Wu}$ et al. [15]; the results showed that compared with the traditional grey model, this model greatly improves the prediction accuracy. Zeng et al. proposed a self-adaptive intelligence grey prediction model with fractional accumulation [16]. Ma et al. proposed a novel definition of conformable fractional accumulation which is more feasible and simple compared with the traditional fractional grey model [17]. Wu et al. further perfected the definition of conformable fractional accumulation and successfully utilized it to predict carbon dioxide emissions of BRICS [18]. Wu et al. utilized a novel fractional nonlinear grey Bernoulli model to forecast short-term renewable energy consumption [19]. Ma et al. proposed a fractional delay grey model based on the grey system optimization algorithm and applied it to the prediction of natural gas consumption in Chongqing [20]. Wu et al. [18] applied the univariate inhomogeneous grey model to study the $\mathrm{CO}_{2}$ emissions of BRICS countries without considering external factors. Under several error metrics, one can see that the grey forecasting model can provide excellent results for the five countries. Based on the definition of the conformable fractional derivative, $\mathrm{Ma}$ et al. [17] proposed conformable fractional accumulation and difference. Then, a novel conformable fractional grey model is proposed based on the fractional accumulation and difference, and the brute force method is introduced to optimize its fractional order.
Fractional grey models are divided into fractional accumulation and derivative grey models. The fractional accumulation generating operator (FAGO) can reflect the characteristics of new information priority and is a generalization of integer accumulation generation [15]. FAGO is extended from a GM $(1,1)$ to grey Bernoulli [21]. The new information priority principle is embodied in the FAGO grey Bernoulli model [22]. To solve the prediction problem with memory characteristics, based on the memory principle, Mao [23] proposed to improve the integral differential equation into a fractional differential equation and established a univariable fractional derivative grey prediction model. Kang [24] extended it to a Caputo-type multivariate fractional grey prediction model. Although some achievements have been made in grey prediction models, they all use first-order differential equations $[25,26]$ or constant fractional differential equations $[23,27]$.

Based on this theoretical knowledge, this paper expands a novel conformable fractional grey model and the optimal weighted combination model to estimate and predict the population of Huizhou of China. The main novelties of this paper can be outlined as follows. Firstly, the fractional grey model and the optimal weighted combination model are compared and analyzed in research of Huizhou's population. Secondly, the optimal conformable fractional order and dynamic background value coefficient are determined by Quantum Inspired Evolutionary Algorithm (QIEA) [28]. In order to verify the effectiveness of the two new models, the two models were compared with GM $(1,1)$ model, regression model, and growth model. Finally, the optimal weighted combination model and the fractional grey model are applied to predict the population of Huizhou of China from 2019 to 2021.

\subsection{Modeling Process of FGM $(1,1)$.}

(1) Construct an additive sequence of order $r$ : Write the original nonnegative data as the original order column $U^{(0)}=\left\{u^{(0)}(1), u^{(0)}(2), \ldots, u^{(0)}(n)\right\}$. After calculation, the rorder accumulation sequence $U^{(r)}=\left\{u^{(r)}(1), u^{(r)}(2), \ldots, u^{(r)}(n)\right\} \quad$ is obtained where $u^{(k)}(r)=\sum_{i=1}^{k} C_{k-i+r-1}^{k-i} u^{(0)}(i), C_{r-1}^{0}=1, C_{k}^{K+1}$ $=0, k=0,1, \ldots, n-1$,

$$
C_{k-i+r-1}^{k-i}=\frac{(k-i+r-1)(k-i+r-2), \ldots,(r+1) r}{(k-i) !} .
$$

(2) Set up the differential equation and solve it: Set up the whitening differential equation as follows:

$$
\frac{\mathrm{d} x^{(r)}(t)}{\mathrm{d} t}+a x^{(r)}(t)=b .
$$

$$
\text { Suppose } s^{(r)}(t)=0.5\left[u^{(r)}(t)+u^{(r)}(t-1)\right], w^{(r)}(k)
$$$$
=\left[u^{(r)}(t)-u^{(r)}(t-1)\right](t=2,3, \ldots, n) \text {. Then, }
$$ 


$$
\begin{aligned}
B & =\left[\begin{array}{cc}
-s^{(r)}(2) & 1 \\
-s^{(r)}(3) & 1 \\
\vdots & \vdots \\
-s^{(r)}(n) & 1
\end{array}\right], \\
D & =\left[\begin{array}{c}
w^{(r)}(2) \\
w^{(r)}(3) \\
\vdots \\
w^{(r)}(n)
\end{array}\right] .
\end{aligned}
$$

Use the least square method to obtain the numerical solution of the parameters as follows:

$$
\left[\begin{array}{l}
\widehat{a} \\
\widehat{b}
\end{array}\right]=\left(B^{T} B\right)^{-1} B^{T} D \text {. }
$$

The formal solution of equation (6) is

$$
\widehat{u}^{(r)}(t+1)=\left(u^{(0)}(1)-\frac{\widehat{b}}{\widehat{a}}\right) e^{-\widehat{a} t}+\frac{\widehat{b}}{\widehat{a}} .
$$

(3) Accumulation and subtraction of $r$-order sequence: Change $U^{(r)}=\left\{\widehat{u}^{(r)}(1), \widehat{u}^{(r)}(2), \ldots, \widehat{u}^{(r)}(n), \ldots\right\}$ is accumulated for order $1-r$ and then accumulated and subtracted for order 1 , which is

$$
\begin{aligned}
\alpha^{(r)} U^{(0)}= & \left\{\alpha^{(1)} \widehat{u}^{(1-r)}(1), \alpha^{(1)} \widehat{u}^{(1-r)}(2), \ldots,\right. \\
& \left.\alpha^{(1)} \widehat{u}^{(1-r)}(n), \alpha^{(1)} \widehat{u}^{(1-r)}(n+1), \ldots\right\} .
\end{aligned}
$$

Calculate the fitted value $\widehat{u}^{(0)}(1), \widehat{u}^{(0)}(2), \ldots, \widehat{u}^{(0)}(n)$ and the predicted value $\widehat{u}^{(0)}(n+1), \widehat{u}^{(0)}(n+2), \ldots$

(4) Evaluation model: The mean absolute percentage error (MAPE) was used to evaluate the model:

$$
\text { MAPE }=\frac{1}{n} \sum_{t=1}^{n}\left|\frac{\left(\widehat{u}_{t}-u_{t}\right)}{u_{t}}\right| .
$$

(5) The optimal order $r$ is selected to get the predicted sequence as follows:

$$
\widehat{u}^{(0)}(n), \widehat{u}^{(0)}(n+1), \ldots, \widehat{u}^{(0)}(n+m) .
$$

(6) Error test: Perform residual test or correlation test or calibration error probability test for $\widehat{u}^{(0)}(1), \widehat{u}^{(0)}(2), \ldots, \widehat{u}^{(0)}(n)$, judge the fitting effect of the model according to the test result, and qualitatively analyze the prediction accuracy of the model; if the fitting accuracy is not ideal, the cumulative order can be adjusted $r$, that is, return to (1) until the accuracy reaches the ideal state.

\section{Empirical Analysis of the Optimal Weighted Combination Model and FGM}

4.1. Linear Regression Model. Since population prediction has certain assumptions and stable population theory is an important prerequisite, choosing a specific linear function or curve function to fit the historical data of population development has a certain impact on short-term population prediction, and the basic data required are less and easy to obtain. The data in this paper are from the statistical yearbook of Huizhou. The permanent resident population data of Huizhou from 2008 to 2018 are selected. The linear regression model is fitted, and SPSS is used for correlation analysis. The $R^{2}$ is 0.769 , so the fitting error is not large.

The linear regression equation is as follows:

$$
y=432.41+5.21 x
$$

The fitting Figure 1 is drawn. See Table 1 for the estimated values in $2008-2018$.

4.2. $G M(1,1)$. On the basis of the fuzzy uncertainty in fuzzy mathematics, the population system is regarded as a grey system and its future development is predicted. The GM model was proposed by Professor Deng [7]. This paper selects the permanent population data of Huizhou from 2008 to 2018 to build an estimated model. Python software was used for modeling and testing. The posterior difference ratio of this model is 0.37535 , which meets the requirement of level 2 accuracy (qualified), and the predicted result is in good fitting degree with the actual situation. The population development of Huizhou from 2019 to 2021 shows a linear trend of growth, which is consistent with the stable mortality rate and recently improved fertility rate of Huizhou. It shows the release of fertility potential energy in recent years due to the influence of policies and economy, and the population growth trend is shown in Figure 1 . The estimated value of $2008-2018$ is shown in Table 1.

4.3. Growth Model. The permanent resident population from 2008 to 2018 was selected from the data block, and the population growth estimation model was established. The model considered the needs of the block growth model based on the previous population growth rate to estimate the regional maximum population capacity and population growth rate, and then sample data were selected to fit the population development trend of Huizhou. In this paper, the growth model is established by using MATLAB software. The modeling steps are as follows:

(1) Establish a function file and import the growth function:

$$
y=e^{\beta_{0}+\beta_{1} \mathrm{x}} .
$$

(2) Import population data.

(3) Select the least square method and fit function in MATLAB; then, the parameter estimation can be completed and the growth model can be obtained.

(4) The regression model is as follows:

$$
y=e^{6.07+0.011 x}
$$

From 2008 to 2018, Huizhou's population growth rate has been on the rise. The overall population shows an 


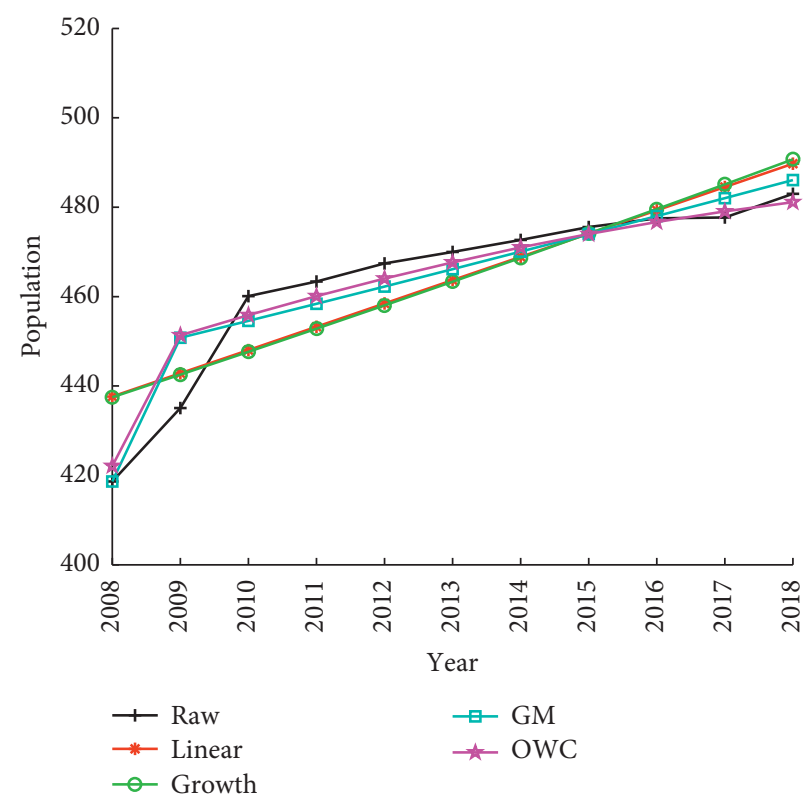

FIGURE 1: Huizhou population estimated results of various models.

TABLE 1: Estimated results of Huizhou population with various models, units: 10,000.

\begin{tabular}{lcccccccc}
\hline Year & Raw & Linear & Growth & GM & OWC & FGM & ARIMA & Logistic \\
\hline 2008 & 418.65 & 437.62 & 437.47 & 418.65 & 422.08 & 418.65 & 436.45 \\
2009 & 435.08 & 442.83 & 442.53 & 450.79 & 451.32 & 436.79 & 432.32 \\
2010 & 460.11 & 448.04 & 447.64 & 454.58 & 455.85 & 452.83 & 455.92 \\
2011 & 463.36 & 453.25 & 452.81 & 458.40 & 460.09 & 462.62 & 472.40 \\
2012 & 467.40 & 458.46 & 458.04 & 462.25 & 464.03 & 468.99 & 465.16 \\
2013 & 470.00 & 463.67 & 463.33 & 466.14 & 467.66 & 473.19 & 464.59 \\
2014 & 472.66 & 468.88 & 468.68 & 470.06 & 470.98 & 475.91 & 462.18 \\
2015 & 475.55 & 474.09 & 474.09 & 474.01 & 473.99 & 477.55 & 470.63 \\
2016 & 477.50 & 479.30 & 479.57 & 478.00 & 476.69 & 478.37 & 477.42 \\
2017 & 477.70 & 484.51 & 485.10 & 482.02 & 479.06 & 478.54 & 484.94 & 463.28 \\
2018 & 483.00 & 489.72 & 490.71 & 486.07 & 481.11 & 478.20 & 489.18 & 479.02 \\
MAPE & & $1.70 \%$ & $1.76 \%$ & $1.03 \%$ & $0.81 \%$ & $0.56 \%$ & 484.64 \\
\hline
\end{tabular}

increasing trend, and the fertility potential energy is still in the release period. By 2021, the population growth tends to 5.0471 million. Draw the fitting Figure 1 is drawn. See Table 1 for the estimated values in 2008-2018.

\subsection{Application of Optimal Weighted Combination (OWC)} Model

(1) Construct the optimal weighted combination model:

$$
\left\{\begin{array}{l}
\min Q=\sum_{i=1}^{m} e_{i t}^{2}=e^{T} e=K^{T} E K \\
\text { s.t. } \sum_{i=1}^{m} k_{i}=R^{T} K=1
\end{array}\right.
$$

(2) Select an error statistic, i.e., fitting error, and calculate the error information matrix.
(3) According to the predicted and actual values of the above three submodels, the fitting error and the prediction error information matrix are calculated:

$$
\begin{aligned}
E & =\left[\begin{array}{ccc}
\sum_{t=1}^{n} e_{1 t}^{2} & \sum_{t=1}^{n} e_{1 t} e_{2 t} & \sum_{t=1}^{n} e_{1 t} e_{3 t} \\
\sum_{t=1}^{n} e_{2 t} e_{1 t} & \sum_{t=1}^{n} e_{2 t}^{2} & \sum_{t=1}^{n} e_{2 t} e_{3 t} \\
\sum_{t=1}^{n} e_{3 t} e_{1 t} & \sum_{t=1}^{n} e_{3 t} e_{2 t} & \sum_{t=1}^{n} e_{3 t}^{2}
\end{array}\right] \\
& =\left[\begin{array}{lll}
898.96 & 921.14 & 372.01 \\
921.14 & 945.40 & 381.45 \\
372.01 & 381.45 & 380.74
\end{array}\right] .
\end{aligned}
$$

(4) Calculate the weight of the model: 
According to the Lagrange multiplier method, the weight coefficient is solved as follows:

$$
K_{0}=\frac{E^{-1} R}{R^{T} E^{-1} R}=\left[\begin{array}{lll}
5.65 & 0.86 & -5.51
\end{array}\right]^{T} .
$$

Therefore, in the optimal weighted combination model, the weight coefficients of each combination are as follows: the linear regression model is 5.65 , the grey prediction model is 0.86 , and the growth model is -5.51 . Therefore, the final prediction model is

$$
Q_{0}=5.65 \hat{y}_{1}+0.86 \hat{y}_{2}-5.51 \widehat{y}_{3} .
$$

The prediction results are shown in Table 1 .

4.5. FGM. Construct the original sequence of population data in Huizhou from 2008 to 2018 (unit: ten thousand) $U^{(0)}=\{418.65,435.08,460.11,463.36,467.40,470.00,472$. $66,475.55,477.50,477.70,483\}$. 0.9 order accumulation sequence is

$$
U^{(0.9)}=\{418.65,811.86,1209.62,1595.46,1974.77,2348.33,2717.72,3084.17,3447.44,3806.39,4166.76\} .
$$

Use the least square method to calculate the unknown where parameters $\widehat{a}$ and $\widehat{b}$ :

$$
\left[\begin{array}{l}
\widehat{a} \\
\widehat{b}
\end{array}\right]=\left(B^{T} B\right)^{-1} B^{T} D=\left(\begin{array}{c}
0.01743 \\
402.16
\end{array}\right),
$$

$$
\begin{aligned}
B^{T} & =-\left[\begin{array}{cccccccccc}
615.25 & 1010.74 & 1402.54 & 1785.12 & 2161.55 & 2533.03 & 2900.95 & 3265.81 & 3626.92 & 3986.58 \\
-1 & -1 & -1 & -1 & -1 & -1 & -1 & -1 & -1 & -1
\end{array}\right], \\
D^{T} & =\left[\begin{array}{llllllllll}
393.21 & 397.76 & 385.83 & 379.30 & 373.55 & 369.39 & 366.45 & 363.26 & 358.95 & 360.36
\end{array}\right] .
\end{aligned}
$$

Obtain the response function between time periods:

The fitting value of order 0.9 additive sequence is

$$
\widehat{u}^{(0.9)}(t+1)=\left(418.65-\frac{40216}{1.1743}\right) e^{-0.01743 t}+\frac{40216}{1.1743} \text {. }
$$

$$
\begin{aligned}
\widehat{U}^{(0.9)} & =\left\{\widehat{u}^{(0.9)}(1), \widehat{u}^{(0.9)}(2), \ldots, \widehat{u}^{(0.9)}(11)\right\} \\
& =10^{3} \times\{0.4187,1.0651,1.6384,2.2019,2.7556,3.2997,3.8344,4.3599,4.8763,5.3838,5.8826\}
\end{aligned}
$$

Restore order list as follows:

$$
\begin{aligned}
\widehat{U}^{(1)} & =\left\{\widehat{u}^{(0.9)(0.1)}(1), \widehat{u}^{(0.9)(0.1)}(2), \ldots, \widehat{u}^{(0.9)(0.1)}(10), \widehat{u}^{(0.9)(0.1)}(11),\right. \\
& =\{418.65,855.44,1308.27,1770.89,2239.88,2713.07,3188.98,3666.53,5101.81,5580.01\} .
\end{aligned}
$$

The simulated value of Huizhou population is

$$
\begin{aligned}
\widehat{U}^{(0)} & =\left\{\widehat{u}^{(0)}(1), \widehat{u}^{(0)}(2), \ldots, \widehat{u}^{(0)}(10), \widehat{u}^{(0)}(11),\right. \\
& =\{418.65,436.79,452.83,462.62,468.99,473.19,475.91,477.55,478.37,478.54,478.20\} .
\end{aligned}
$$


Taking the minimum average absolute percentage error value of the FGM $(1,1)$ model as the object function and using the particle swarm optimization algorithm, the prediction effect of the FGM $(1,1)$ model is the best when the order $r$ is 0.9 , and the prediction result is more accurate. Python software is used to complete the above estimation and simulation. The estimated values with fractional grey model are shown in Figure 2.

As can be seen from Figure 1, the optimal weighted combination model balances the characteristics of higher predicted value of the growth model, strong linear trend and rapid growth of the regression model's forecast curve, and the GM $(1,1)$ model has a high degree of agreement with the prediction curve of the optimal weighted combination method. The estimated curves of the optimal weighted combination model are closer to the truth than those of the three submodels. It can be seen from Figure 1 that the truth estimation of the optimal weighted combination model is better than the modification of the three submodels. The value of MAPE in Table 1 can also draw OWC superior to its submodels. The optimal weighted group method predicts that the permanent population of Huizhou will continue to grow and maintain a stable growth from 2019 to 2021. It can be seen that the population scale development trend of Huizhou in the next decade is as follows: under the influence of the national fertility policy reform, the fertility level of Huizhou has been improved, and the permanent resident population of Huizhou will gradually rise in the next decade. By 2021, the total population of Huizhou will reach 5029000, with an average annual growth of $1.49 \%$ and an average annual growth of 72633 . The predicted population values for 2019-2021 are shown in Table 2. Figure 2 shows that FGM estimates the raw data better than OWC. Most points on the FGM curve are closer to the true value than those on the OWC curve. These can be indirectly reflected from the values of MAE and RMSE in Table 2. Figure 2 shows that the fractional grey model has a better fitting effect on the truth value than that of the optimal combination model.

From Table 1, we also get MAPE $=0.56 \%$ of the fractional cumulative grey model, which is smaller than that of the other three methods. The MAPE value of model OWC is smaller than that of its three submodels. At the same time, the MAPE value of OWC is smaller than that of ARIMA model and logistic model.

4.6. Model Test. Seen the values of MAPE from Table 1, the optimal weighted combination model is superior to each submodel. However, the estimation result of the fractional grey prediction model is better than that of the optimal weighted combination model. To further compare the estimated results of these two models, we apply the mean absolute error and root mean square error to measure them.

Mean absolute error (MAE) is as follows:

$$
\text { MAE }=\frac{1}{N} \sum_{t=1}^{n}\left|\left(\hat{y}_{t}-y_{t}\right)\right| \text {. }
$$

Root mean square error (RMSE) is as follows:

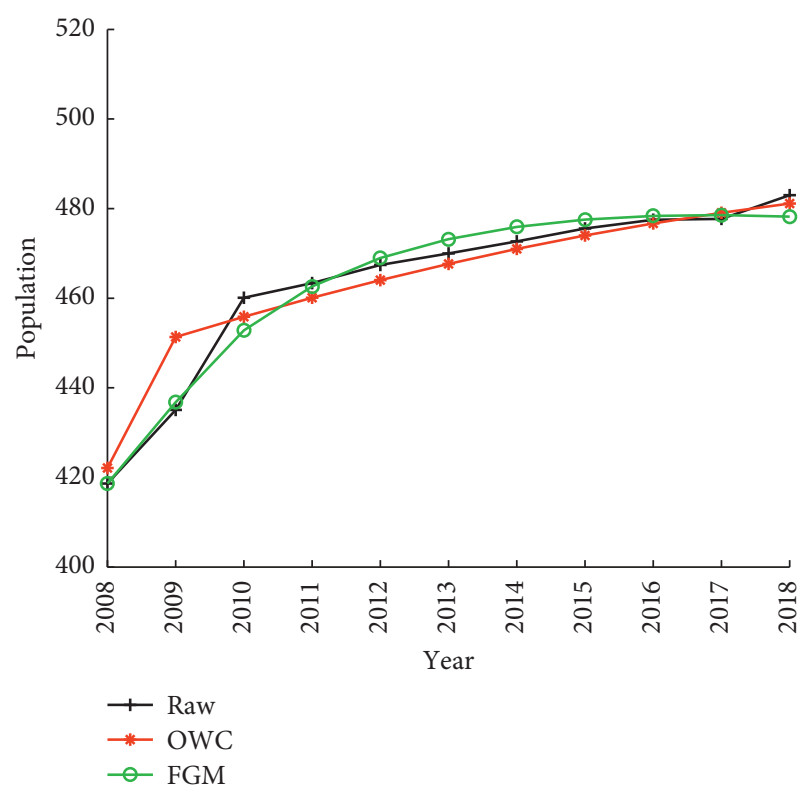

FIgURE 2: Comparison graph of Huizhou population development trend estimated by the optimal weighted group model and the fractional grey model.

TABLE 2: Test results of the prediction model.

\begin{tabular}{lcccc}
\hline Year & OWC & FGM & ARIMA & Logistic \\
\hline 2019 & 497.42 & 477.43 & 499.21 & 494.99 \\
2020 & 500.31 & 476.31 & 512.83 & 500.14 \\
2021 & 502.90 & 474.90 & 520.77 & 505.27 \\
MAE & 4.1334 & 2.3882 & 6.7958 & 7.6208 \\
RMSE & 5.9247 & 3.1369 & 8.2047 & 8.8579 \\
\hline
\end{tabular}

$$
\mathrm{RMSE}=\sqrt{\frac{1}{N} \sum_{t=1}^{n}\left(\hat{y}_{t}-y_{t}\right)^{2},}
$$

where $N$ is the total number of years, $y_{t}$ is the real value, and $\hat{y}_{t}$ is the estimated value.

Table 2 shows that the MAE of the OWC model is 4.1334, and the MAPE is 5.9247, and the MAE of FGM is 2.3882, and RMSE is 3.1369. We add ARIMA and logistic to estimate and forecast the original data and find that MAE and RMSE values of these two methods are larger than those of OWC and FGM. The results show that the prediction effect of the optimal weighted combination model is obviously poor than that of the fractional grey model. However, when it comes to predicting trends, the optimal weighting combination is clearly stronger than that of the fractional grey model. The population of Huizhou has been increasing steadily in recent years. From the perspective of stability, when the sample size is small, the fractional grey model is relatively stable.

\section{Conclusions and Suggestions}

The present situation of population development in Huizhou city is analyzed qualitatively and quantitatively. Then, we 
adopted a series of mathematical methods, i.e., the fractional grey model, the optimal weighted combination method, the linear regression model, the growth model, and the GM (1, 1) prediction model, combined with MATLAB and Python to forecast the population development trend of Huizhou and analyzed the predicted results. We can obtain that the total population development trend of Huizhou is increasing year by year from 2019 to 2021 . It will break through 5 million population marks around the end of 2021. In the estimation and prediction of Huizhou population, the optimal combination model and the fractional grey model have their advantages. In the next decade, the population of Huizhou will increase steadily, the population growth rate will be slower over time, but there will be no downward trend. According to the above conclusions, we proposed the following two suggestions: on the one hand, we will continue to implement the national three-child policy, increase subsidies for family planning, and raise the fertility level; on the other hand, measures should be taken to combine family care with social care to ensure that the elderly can rely on others for retirement and support.

In this paper, we combine the optimal weighted combination method and the fractional grey model to implement the prediction of the total population development trend of Huizhou in three years and analyze the results in depth. The forecast results show that the total population of Huizhou city will increase year by year from 2019 to 2021, and the labor supply is relatively sufficient in a demographic dividend period. However, it will soon enter a small and aging population, which is not conducive to this sustainable development. Therefore, we must pay attention to the population planning of Huizhou and turn the population advantage into the capital advantage in a period of demographic dividend. At the same time, we will raise the fertility rate and improve the old-age security system. Our future work is to study the population of Huizhou with the novel conformable fractional nonhomogeneous grey model and nonhomogenous discrete grey model with fractionalorder accumulation.

\section{Data Availability}

The data used to support the findings of this study are included within the article.

\section{Conflicts of Interest}

The authors declare that they have no conflicts of interest.

\section{Acknowledgments}

This work was supported by the NSF of Fujian Province, PR China (2020J01170), Fujian Normal University Innovation Team Foundation, PR China (IRTL1704), "Hundred Excellent Young Teachers Training Project" of Huizhou University projects from NSF of Huizhou University (Grant No. hzu201806), and Huizhou University School-Level Undergraduate Teaching Quality Engineering Project (XJYJG2021045, 15109038).

\section{References}

[1] X. M. Ni, X. X. Shen, S. Hung, and J. Y. Zhang, "The convergence trend of the age structure and the aging population in China," Journal of Applied Sport Management, vol. 39, no. 2, pp. 191-205, 2020.

[2] Y. Zeng, "Past, present and future of demography," Population Research, vol. 33, no. 5, pp. 8-22, 2009.

[3] M. .Z. Luo, C. H. Gan, X. W. Huang, and W. L. Zhou, "Shift from government paradigm to governance paradigm: a case analysis of the comprehensive reform in family planning in Huizhou," South China Population, vol. 26, no. 2, pp. 13-19, 2011.

[4] J. Song, "Inheritance and evolution of demographic methods: and on the development of demography in China," Population \& Economics, vol. 1, pp. 1-14, 2020.

[5] R. C. Zha, "Several basic problems of population prediction," Population Research, vol. 2, pp. 25-32, 1980.

[6] J. Song, J. Y. Yu, and D. Y. Kong, "Population control theory," China Soft Science, vol. 1, pp. 1-7, 1989.

[7] J. L. Deng, "Gray control system," Journal of Central China Institute of Technology, vol. 3, pp. 9-18, 1982.

[8] Y. H. Chen, "Child age progressive population development model," China Population Science, vol. 6, pp. 29-34, 1994.

[9] Y. H. Hao and X. M. Wang, "The dynamic model of gray system and its application to population forecasting," Mathematics in Practice and Theory, vol. 5, pp. 813-820, 2002.

[10] Z. W. Zhai, L. Li, J. J. Chen, and W. Chen, "Applications of population projection in the PADIS-INT: comparative study on MORTPAK," Spectrum and PADIS-INT, Population Research, vol. 41, no. 6, pp. 84-97, 2017.

[11] G. Z. Wang, "China's population forecast methods and future population policies," Financial Minds, vol. 3, no. 3, pp. 112138, 2018.

[12] Y. Shang and Y. M. Ding, "Evaluation and analysis of optimal combination forecasting methods," Statistics \& Decisions, vol. 17, pp. 122-123, 2005.

[13] K. Y. Mao, Determination and Application of Weight in Combination Forecasting, pp. 9-16, Chengdu University of Technology, Sichuan, China, 2007.

[14] X. W. Tang, "Optimal combination forecasting method and its application," Journal of Applied Sport Management, vol. 1, pp. 31-35, 1992.

[15] L. Wu, S. Liu, L. Yao, S. Yan, and D. Liu, "Grey system model with the fractional order accumulation," Communications in Nonlinear Science and Numerical Simulation, vol. 18, no. 7, pp. 1775-1785, 2013.

[16] B. Zeng and S. Liu, "A self-adaptive intelligence gray prediction model with the optimal fractional order accumulating operator and its application," Mathematical Methods in the Applied Sciences, vol. 40, no. 18, pp. 7843-7857, 2017.

[17] X. Ma, W. Wu, B. Zeng, Y. Wang, and X. Wu, "The conformable fractional grey system model," ISA Transactions, vol. 96, pp. 255-271, 2020.

[18] W. Wu, X. Ma, Y. Zhang, W Li, and Y Wang, "A novel conformable fractional non-homogeneous grey model for forecasting carbon dioxide emissions of BRICS countries," The Science of the Total Environment, vol. 707, Article ID 135447, 2020.

[19] W. Wu, X. Ma, B. Zeng, Y. Wang, and W. Cai, "Forecasting short-term renewable energy consumption of China using a novel fractional nonlinear grey Bernoulli model," Renewable Energy, vol. 140, pp. 70-87, 2019. 
[20] X. Ma, X. Mei, W. Wu, X. Wu, and B. Zeng, "A novel fractional time delayed grey model with Grey Wolf Optimizer and its applications in forecasting the natural gas and coal consumption in Chongqing China," Energy, vol. 178, pp. 487-507, 2019.

[21] Q. Xiao, M. Shan, M. Gao, X. Xiao, and M. Goh, "Parameter optimization for nonlinear grey Bernoulli model on biomass energy consumption prediction," Applied Soft Computing, vol. 95, Article ID 106538, 2020.

[22] Y. Zhang, S. Mao, and Y. Kang, "A clean energy forecasting model based on artificial intelligence and fractional derivative grey Bernoulli models," Grey Systems: Theory and Application (ahead-of-print), vol. 11, no. 4, pp. 571-595, 2021.

[23] S. H. Mao, M. Y. Gao, X. P. Xiao, and M. Zhu, "A novel fractional grey system model and its application," Applied Mathematical Modelling, vol. 40, no. 7-8, pp. 5063-5076, 2016.

[24] Y. X. Kang, S. .H. Mao, and Y. H. Zhang, "Fractional derivative multivariable grey model for nonstationary sequence and its application," Journal of Systems Engineering and Electronics, vol. 31, no. 5, pp. 1011-1020, 2020.

[25] H. Duan, X. Xiao, J. Long, and Y. Liu, "Tensor alternating least squares grey model and its application to short-term traffic flows," Applied Soft Computing, vol. 89, Article ID 106145, 2020.

[26] S. Mao, M. Zhu, X. Wang, and X. Xiao, "Grey-Lotka-Volterra model for the competition and cooperation between thirdparty online payment systems and online banking in China," Applied Soft Computing, vol. 95, Article ID 106501, 2020.

[27] Y. Zhang, S. Mao, Y. Kang, and J. Wen, "Fractal derivative fractional grey Riccati model and its application," Chaos, Solitons \& Fractals, vol. 145, Article ID 110778, 2021.

[28] K. Kuk-Hyun Han and J. Jong-Hwan Kim, "Quantum-inspired evolutionary algorithm for a class of combinatorial optimization," IEEE Transactions on Evolutionary Computation, vol. 6, no. 6, pp. 580-593, 2002. 